\title{
Electromagnetic Scattering from arbitrary flat plates: Analysis of the problem by using Method of Moments with different sinc type basis functions
}

\author{
$\underline{\text { Basak Ozbakis }}^{1}$, Taner Oguzer $^{2}$, and Alp Kustepeli ${ }^{1}$ \\ ${ }^{1}$ Izmir Institute of Technology, Electrical and Electronics Engineering 35430 Urla, Izmir, Turkey. \\ basakozbakis@iyte.edu.tr \\ alpkustepeli@iyte.edu.tr \\ ${ }^{2}$ Dokuz Eylul University, Electrical and Electronics Engineering 35160 Tinaztepe-Buca, Izmir, Turkey. \\ taner.oguzer@deu.edu.tr
}

\begin{abstract}
In this paper, three dimensional electromagnetic scattering problem is solved by using pulse-sinc type basis functions in the Method of Moments $(\mathrm{MoM})$ procedure. This method is applied to the scattering problems of a plane wave illuminated flat arbitrary geometries. Pulse-sinc based MoM formulation is developed, the current densities and radar cross section of different geometries are investigated. The radar cross section (RCS) for co-polarized and cross polarized cases of the flat plate geometries are compared with both of the results obtained from the sinc-sinc based formulation and SuperNEC. The results obtained by using pulse-sinc fornulation are in very good agreement with those of the SuperNEC.
\end{abstract}

\section{Introduction}

Method of Moments is generally used for the numerical solution of the electromagnetic scattering problems [1]. In the application of this method, different kinds of basis functions, such as entire domain and subdomain basis functions, are employed for the expansion of the unknown current $[2,3]$.

In the literature, sinc functions are also used to solve integral equations (IEs), ordinary and partial differential equations that characterize the initial and boundary value problems [4]. The important analytical properties of the sinc functions are used in the formulation. Sinc functions are defined as quasi-localized band-limited basis functions in [5] and easily implemented in the problems having singularities. The computational burden is reduced by their application in some linear algebraic equations [6]. The use of sinc based MoM formulation in 2D scattering problems is investigated in [7-9]. The method has been extended to 3D problems in our previous study by using sinc-sinc based formulation and the results are compared with the ones obtained from the rooftop basis based formulation [10].

In this study, 3D scattering problems are investigated by using pulse-sinc type basis and testing functions to avoid the overflow problem at the edges. The number of integrals to be computed is again decreased by using sinc functions and as a result of this, the CPU time for obtaining MoM matrix elements is decreased. The RCS results for copolarized and cross polarized cases based on pulse-sinc formulation are compared with those of the sinc-sinc based MoM formulation that is obtained in [10] and with the ones obtained from SuperNEC. It is observed that pulse-sinc formulation increases the accuracy in the results.

\section{Formulation}

The components of the electric field can be determined from

$$
\mathbf{E}=-j \omega \mathbf{A}-\nabla \Phi
$$

where $\mathbf{A}$ and $\Phi$ are magnetic vector and electric scalar potential respectively. In (1), $\mathbf{A}$ and $\Phi$ can be obtained by using the convolution of the current density with the Green's function and the convolution of the charge density, $\rho=-\nabla \cdot \mathbf{J} / j \omega$, with the Green's function, respectively. The $\mathrm{x}$ and $\mathrm{y}$ components of the scattered field are given by 


$$
\begin{aligned}
& E_{x}=-j \omega \mu\left[J_{x} * G\right]+\frac{1}{j \omega \varepsilon} \frac{\partial}{\partial x}\left[\frac{\partial J_{x}}{\partial x^{\prime}} * G\right]+\frac{1}{j \omega \varepsilon} \frac{\partial}{\partial x}\left[\frac{\partial J_{y}}{\partial y^{\prime}} * G\right] \\
& E_{y}=-j \omega \mu\left[J_{y} * G\right]+\frac{1}{j \omega \varepsilon} \frac{\partial}{\partial y}\left[\frac{\partial J_{y}}{\partial y^{\prime}} * G\right]+\frac{1}{j \omega \varepsilon} \frac{\partial}{\partial y}\left[\frac{\partial J_{x}}{\partial x^{\prime}} * G\right]
\end{aligned}
$$

where the Green's function is $G\left(\vec{r}-\vec{r}^{\prime}\right)=\frac{e^{-j k R}}{4 \pi R}, R=\sqrt{\left(x-x^{\prime}\right)^{2}+\left(y-y^{\prime}\right)^{2}+\left(z-z^{\prime}\right)^{2}}$. The current densities are expanded in terms of the basis functions such as

$$
\begin{aligned}
& J_{x}=\sum_{s=1}^{N} a_{s} \underbrace{\operatorname{sinc}\left(2 W_{x} x-p(s)\right) \operatorname{pulse}\left(y, q(s), t_{y}\right)}_{B_{x n(s)}(x, y)} \\
& J_{y}=\sum_{k=1}^{M} b_{k} \underbrace{\operatorname{sinc}\left(2 W_{y} y-\bar{q}(k)\right) \operatorname{pulse}\left(x, \bar{p}(k), t_{x}\right)}_{B_{y n(k)}(x, y)}
\end{aligned}
$$

where $N$ and $M$ are the numbers of sampling points for $x$ and $y$ directed currents. The bandwidths of the sinc functions are $W_{x}$ and $W_{y}$ and they determine the position of the function in $x$ and $y$ directions as $p(s) / 2 W_{x}$ and $\bar{q}(k) / 2 W_{y}$. Pulse functions in $x$ and $y$ directions are located at the point $\bar{p}(k) t_{x}-t_{x} / 2$. and $q(s) t_{y}-t_{y} / 2$, where $t_{x}=1 / 2 W_{x}$ and $t_{y}=1 / 2 W_{y}$.

Galerkin method is employed to obtain the MoM matrix elements. The convolution between basis function and Green's function is given by

$$
B_{x n(s)}\left(x^{\prime}, y^{\prime}\right) * G\left(x-x^{\prime}, y-y^{\prime}\right)=\underbrace{\int_{q t_{y}-t_{y} / 2}^{q t_{y}+t_{y} / 2} \int_{-\infty}^{\infty} \operatorname{sinc}\left(2 W_{x} x^{\prime}-p(s)\right) \operatorname{pulse}\left(y^{\prime}, q(s), t_{y}\right) G\left(x-x^{\prime}, y-y^{\prime}\right) d x^{\prime} d y^{\prime}}_{H_{x}(x, y, p(s), q(s))}
$$

where $H_{x}$ can be expanded in a double series with the coefficients $h_{s}^{o}$. The procedure is same for $H_{y}(x, y, \bar{p}(k), \bar{q}(k))$ that can be expanded with $\bar{h}_{s}^{o}$ coefficients similarly. The coefficients $h_{s}^{o}$ and $\bar{h}_{s}^{o}$ can be obtained by taking the inverse of the $H_{x}$ and $H_{y}$ functions using complete orthogonal set of sinc functions. The differentiation is taken over $H_{x}$ and $H_{y}$ numerically by using finite difference method. Similar procedure is employed for testing to obtain the coefficient matrix elements. The singularity has the form $1 / R$ and it is extracted and integrated analytically.

\section{Numerical Results}

In this section, radar cross section simulation results based on pulse-sinc based formulation are compared with those of the sinc-sinc based formulation and they are also compared with the results obtained from SuperNEC. The geometry and the results for a $2 \lambda \times 2 \lambda$ square flat plate are shown in Figure 2. The radar cross sections are also presented in the Figure 2 and they are computed for bistatic case. The RCS results are obtained for cross-polarized (vertical-horizontal) and co polarized (vertical-vertical) cases. The plate is excited by a plane wave with incident angles $\theta^{i n}=45^{\circ}$ and $\varphi^{i n}=0^{\circ}$, while the observation angle, $\varphi^{o b s}$, is $45^{\circ}$ and $90^{\circ}$ for $2 \lambda \times 2 \lambda$ square flat plate. In the computations, 15 unknowns are used for each wavelength. If the Figure 2 is examined one can see that the results obtained from the pulse-sinc formulation are more accurate than those obtained from the sinc-sinc formulation when compared to the ones obtained from the SuperNEC. Simulations are carried out on a Intel(R) Pentium(R)M CPU 1.6 


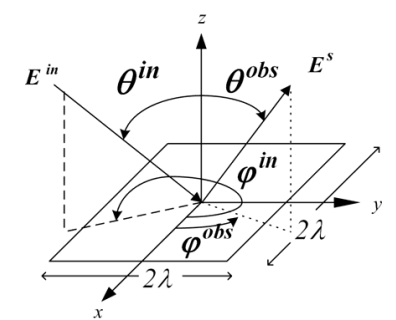

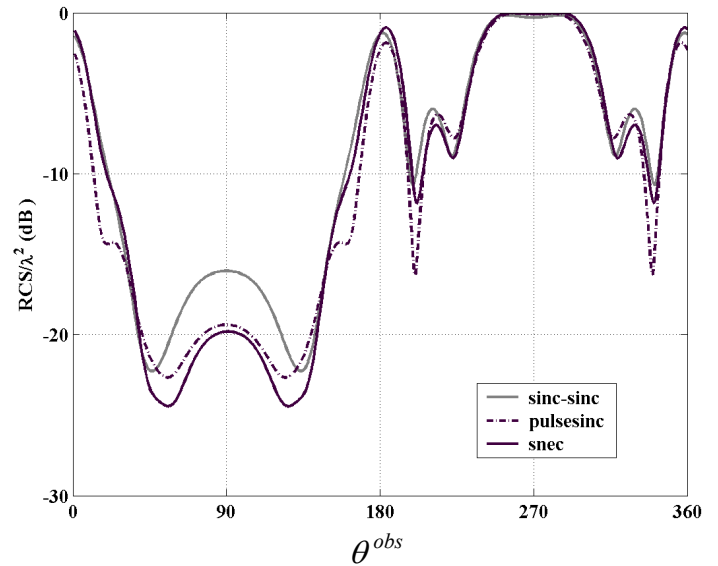

(a)

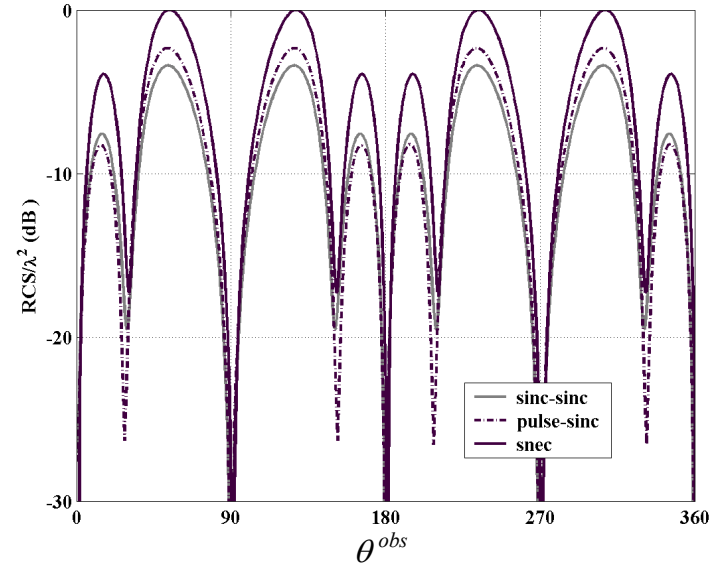

(b)

Figure 2. Normalized radar cross section for a square flat plate $2 \lambda \times 2 \lambda, \theta^{i n}=45^{\circ}$ and $\varphi^{i n}=0^{\circ}$. (a) vertical-horizontal, $\varphi^{o b s}=45^{\circ}(\mathrm{b})$ vertical-vertical, $\varphi^{o b s}=90^{\circ}$.
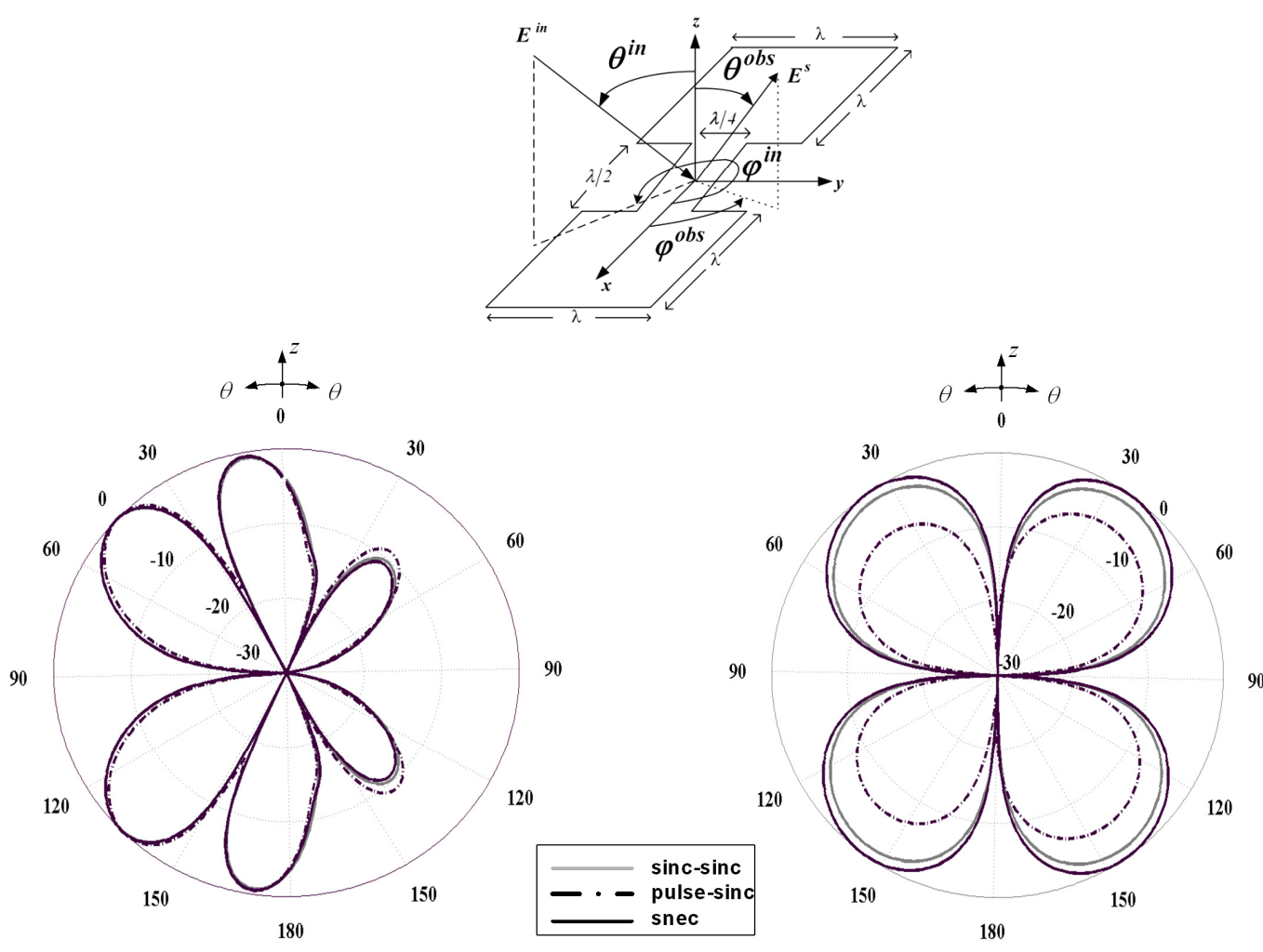

$\varphi^{o b s}=30^{\circ}$

$\varphi^{o b s}=90^{\circ}$

Figure 3. Normalized radar cross section for an arbitrary flat plate geometry (vertical-vertical), $\varphi^{i n}=0^{\circ}, \theta^{i n}=45^{\circ}$. 
$\mathrm{GHz}$ processor with $496 \mathrm{MB}$ of RAM under Fortran90 program packages on the windows operating systems. SuperNEC 2.7 is used for the comparisons. The matrix filling time for the fortran programs based on pulse-sinc and sinc-sinc is 1.48 seconds and 1.46 seconds respectively while the filling time of the SuperNEC is 12.07 seconds for a $2 \lambda \times 2 \lambda$ square flat plate coefficient matrix. An arbitrary flat plate is the second geometry to be examined in this study. The similar comparisons are made and similar results are also obtained for this case. The dimensions of the arbitrary plate are about $2.5 \lambda \times \lambda$ as shown in Figure 3. The plate is excited with a plane wave with incident angles $\theta^{i n}=45^{\circ}$ and $\varphi^{i n}=0^{\circ}$. Radar cross section is observed at the angles, $\varphi^{\text {obs }}, 30^{\circ}$ and $90^{\circ}$. In these simulations, 16 unknowns are taken for each wavelength. Good agreements realized in all the above figures indicates that our pulse-sinc based MoM gives accurate results for flat plate geometries.

\section{Conclusion}

In this study, pulse-sinc based basis functions are used in MoM for the electromagnetic scattering by arbitrarily shaped flat plate surfaces. Pulse-sinc based formulation is developed and then the accuracy of the fortran program is tested with simulations. The simulation results are given for the $2 \lambda \times 2 \lambda$ square flat plate and $2.5 \lambda \times \lambda$ arbitrary flat plate. Pulse-sinc based simulation results are compared with those of the sinc-sinc based MoM and with the ones obtained from SuperNEC. Radar cross sections for co-polarized and cross polarized cases are compared for the two different geometries each other. According to the simulation results, one can obtain that the overflow problem at the edges can be decreased by using pulse functions. The comparisons demonstrate that the results obtained from the pulsesinc based MoM give accurate results compared to the sinc-sinc based results in some cases. The CPU in filling the MoM matrix elements is decreased by reducing the number of integrals to be computed. Furthermore, both results of the sinc based MoM formulations, pulse-sinc and sinc-sinc, give similar results with SuperNEC generally, although their formulations are different. In conclusion, it is observed that in some cases pulse-sinc formulation increases the accuracy in the results.

\section{References}

1. R. F. Harrington, Field Computation by Moment Methods, IEEE Press, 1993.

2. A. F. Peterson, S. L. Ray and R. Mittra, Computational Methods for Electromagnetics, IEEE Press, 1998.

3. S. M. Rao, D. R. Wilton and A. W. Glisson, "Electromagnetic Scattering by Surfaces of Arbitrary Shape", IEEE Trans. on Antennas Propagation, vol. AP-30, no.3, May 1982, pp. 409-418.

4. F. Stenger, Numerical Methods based on Sinc and Analytic Functions, SpringerVerlag, 1993.

5. G. F. Herrmann, "Note on interpolational Basis Functions in the Method of Moments," IEEE Trans. on Antennas and Propagation, vol.38, no.1, January 1990.

6. A. Saadatmandi, M. Razzaghi and M. Dehghan, "Sinc-Collocation methods for the solution of Hallen's integral equation”, J. of Electromagn. Waves and Appl., vol.19, no.2, 2005, pp.245-256.

7. E. X. Huang and A. K. Fung, "An Application of Sampling Theorem to Moment Method Simulation in Surface Scattering," J. of Electromagn. Waves and Appl., vol.20, no.4, 2006, pp. 531-546.

8. T. Oguzer and F. Kuyucuoglu, "Moment of Methods Solution by using Sinc-type Basis Functions for the Scattering from a Finite Number of Conducting Strip Gratings," Turk. J. Elec. Engin., vol.16, no.2, 2008, TUBITAK.

9. T. Oguzer, F. Kuyucuoglu and I. Avgin, "Electromagnetic Scattering from layered strip geometries: The method of moments study with the sinc basis", Turk. J. Elec. Engin., TUBITAK, accepted but not published..

10. B. Ozbakis, T. Oguzer and A. Kustepeli, "Three dimensional Electromagnetic Scattering from the Flat Plates by Using Sinc type Basis Functions in MoM", Computational Electromagnetics International Workshop, CEM 2009, Izmir. 